INPLASY PROTOCOL

To cite: Daveronas et al. Effects of exercise interventions on respiratory drive (Emgdi\%max) in patients with chronic obstructive pulmonary disease: a Systematic Review and Meta-Analysis. Inplasy protocol 202070021. doi: 10.37766/inplasy2020.7.0021

Received: 07 July 2020

Published: 07 July 2020

Corresponding author: Athanasios Daveronas

athanasiosdaveronas@gmail.com

Author Affiliation:

National and Kapodistrian

University of Athens

Support: No funding.

Review Stage at time of this submission: Data analysis.

Conflicts of interest:

The authors declare that they have no conflicts of interest.

\section{Effects of exercise interventions on respiratory drive (Emgdi\%max) in patients with chronic obstructive pulmonary disease: a Systematic Review and Meta-Analysis}

Daveronas, A1; Dinas, P2; Chryssanthopoulos, C3; Nanas, S4; Philippou, $A^{5}$.

Review question / Objective: Do exercise interventions affect respiratory drive (Emgdi\%max) in patients with chronic obstructive pulmonary disease?

Condition being studied: Chronic obstructive pulmonary disease (COPD) is characterized as a common, preventable and treatable disease that is accompanied by persistent respiratory symptoms and airflow limitation that is a result of airway and/or alveolar abnormalities, usually triggered by significant exposure to noxious particles or gases. In COPD patients, dyspnea intensity during exercise correlates strongly with respiratory drive as judged by the amplitude of electromyogram (EMG) in diaphragm expressed in volitional maximum (EMGdi\%max) and can be decreased by exercise interventions.

INPLASY registration number: This protocol was registered with the International Platform of Registered Systematic Review and Meta-Analysis Protocols (INPLASY) on 07 July 2020 and was last updated on 07 July 2020 (registration number INPLASY202070021).

\section{INTRODUCTION}

Review question / Objective: Do exercise interventions affect respiratory drive (Emgdi\%max) in patients with chronic obstructive pulmonary disease?
Condition being studied: Chronic obstructive pulmonary disease (COPD) is characterized as a common, preventable and treatable disease that is accompanied by persistent respiratory symptoms and airflow limitation that is a result of airway and/or alveolar abnormalities, usually 
triggered by significant exposure to noxious particles or gases. In COPD patients, dyspnea intensity during exercise correlates strongly with respiratory drive as judged by the amplitude of electromyogram (EMG) in diaphragm expressed in volitional maximum (EMGdi\%max) and can be decreased by exercise interventions.

\section{METHODS}

Search strategy: The literature databases Pubmed, Pedro, Science direct, and Cochrane Central Register of Controlled Trials were searched from the day of their inception until March 24th, 2020. Any inconsistencies in the searching procedure were resolved by consensus. The terms included in the review: copd; chronic obstructive pulmonary disease; exercise; breathing exercises; aerobic exercise; cardiopulmonary exercise; physical exercise; intensity exercise; eccentric exercise; resistance exercise; intermittent exercise; continuous exercise; exercise based rehabilitation; exercise based rehabilitation; low intensity exercise; low intensity exercise; exercise training; exercise training; respiratory muscle training; respiratory muscle training; strength training; muscle training; resistance training; high intensity interval training; HIIT; HIT; interval training; aerobic training; endurance training; inspiratory muscle training; plyometric training; weight training; physical training; walking; swimming[Title/Abstract]) OR running; cycling[Title/Abstract]); dancing; electrical stimulation; diaphragm electrical stimulation; diaphragm electrical stimulation; electrical muscle stimulation; high intensity exercise; physical endurance; respiratory drive; ventilatory drive; neural respiratory drive; central respiratory drive; neural drive; EMGdi; EAdi; diaphragm EMG; animals; humans.

Participant or population: Patients with chronic obstructive pulmonary disease.

Intervention: Intervention of interest was exercise and training modalities were: a) Aerobic exercise: walking; swimming; running; cycling; dancing, b)resistance exercise: circuit weight training; machine weights; cuff weights; multi-station machine; free weights; pneumaticresistance training equipment; latex resistance bands; calisthenics; bodyweight exercises; ball exercises; body weight exercises, c)respiratory muscle training: inspiratory muscle training; expiratory muscle training; respiratory muscle training devices; respiratory electrical muscle stimulation; diaphragm electrical stimulation.

Comparator: Comparisons between two or more types of exercise (randomized crossover trials) and/or between exercise intervention and control condition (nonrandomized controlled trials).

Study designs to be included: Randomised controlled trials and non-randomised controlled trials.

Eligibility criteria: We included studies that met the following criteria: 1) participants with COPD, 2) human subject research, 3)the studies were published in English, 3)no limit was placed with regard to time of publication, 4)acute or training intervention, 5)comparisons between two or more types of exercise (randomized crossover trials) and/or between exercise intervention and control condition (non-randomized controlled trials), 6)intervention of interest was exercise and 7) training modalities were: a)Aerobic exercise: walking; swimming; running; cycling; dancing, b)resistance exercise: circuit weight training; machine weights; cuff weights; multi-station machine; free weights; pneumatic-resistance training equipment; latex resistance bands; calisthenics; bodyweight exercises; ball exercises; body weight exercises, c)respiratory muscle training: inspiratory muscle training; expiratory muscle training; respiratory muscle training devices; respiratory electrical muscle stimulation; diaphragm electrical stimulation. We excluded studies that were: 1) reviews, 2) grey literature and 3) trials with pharmacological or surgical or "Mechanical Ventilation" intervention. 
Information sources: The literature databases Pubmed, Pedro, Science direct, and Cochrane Central Register of Controlled Trials were searched from the day of their inception until March 24th, 2020.

Main outcome(s): Respiratory drive: the amplitude of electromyogram (EMG) in diaphragm expressed in volitional maximum (EMGdi\%max).

Quality assessment / Risk of bias analysis: The evaluation of study quality was independently performed by two investigators, and inconsistencies were resolved by consensus. The quality of the included studies was assessed by the PEDro Methodological Quality Scale. This 11-item scale evaluates (internal and external) validity and interpretability of studies and can spot potential bias with good reliability. The score is calculated as the sum of the scores for items, apart from the first item.

Strategy of data synthesis: This systematic review was carried out in accordance with PRISMA (Preferred Reporting Items for Systematic Reviews and Meta-Analyses) recommendations.

Subgroup analysis: Not applicable.

Sensibility analysis: Not applicable.

Country(ies) involved: Greece.

Keywords: COPD; Exercise; Neural respiratory drive; Respiratory drive; diaphragm EMG; EMGdi\%max.

Contributions of each author:

Author 1 - Athanasios Daveronas Literature search, Data collection, Study design, Analysis of data, Manuscript preparation.

Author 2 - Petros C. Dinas - Analysis of data, Manuscript preparation, Review of manuscript.

Author 3 - Costas Chryssanthopoulos Manuscript preparation, Review of manuscript.
Author 4 - Serafim Nanas - Manuscript preparation, Review of manuscript.

Author 5 - Anastassios Philippou Literature search, Manuscript preparation, Review of manuscript. 